\title{
Assessing consistency of fish survey data: uncertainties in the estimation of mackerel icefish (Champsocephalus gunnari) abundance at South Georgia
}

\author{
Published in Polar Biology (2015) - DOI 10.1007/s00300-015-1810-0 \\ Fallon, Niall G. ${ }^{1}$; Collins, Martin A. ${ }^{2}$; Marshall, C. Tara ${ }^{1}$; Fernandes, Paul G. ${ }^{1}$ \\ ${ }^{1}$ Institute of Biological and Environmental Sciences, University of Aberdeen, Aberdeen, AB24 2TZ, UK \\ ${ }^{2}$ Government of South Georgia and the South Sandwich Islands Government House, Stanley, Falkland Islands FIQQ 1ZZ, UK \\ Corresponding author: Niall G. Fallon, Institute of Biological and Environmental Sciences, University of Aberdeen, Aberdeen, AB24 2TZ, UK. \\ Email:n.fallon@abdn.ac.uk
}

\begin{abstract}
Mackerel icefish (Champsocephalus gunnari) is a semi-pelagic finfish species inhabiting shelf areas in the Southern Ocean. The population at South Georgia is currently exploited by pelagic trawlers fishing close to the seabed. Annual catches peaked at $150,000 \mathrm{t}$ in 1983 , and have declined since the mid-to-late 1980s. Bottom-trawl surveys have been conducted since 1987, providing a time series of abundance and size distribution for use in assessing the status of the stock and setting quotas. Food-web models suggest that estimates of the biomass from survey data are substantially lower than the amount of icefish required by the local ecosystem. The aim of this study was to assess the uncertainty around current estimates of density and variance, using alternative non-parametric stratified bootstrapping methods. The stratified rescaling bootstrap estimator was identified as the most appropriate method of those tested: in comparison with the existing method, confidence intervals and the interannual variability of the estimates were reduced. Numbers-at-age were estimated from mixture distribution models fitted to length-disaggregated density data in order to determine whether individual cohorts were consistently detected by the
\end{abstract}

surveys. Estimates of numbers-at-age could not consistently delineate cohorts in successive years indicating that survey-based estimates of density were biased. These biases may have arisen because the trawl gear did not select individuals of all sizes equally, or because sampling was restricted to the demersal component of the stock. Estimates of abundance of the pelagic component of the stock should be derived from acoustic data to improve the assessment.

Keywords: mackerel icefish, trawl survey, stratified bootstrap

\section{Introduction}

Mackerel icefish (Champsocephalus gunnari) is a semi-pelagic finfish species occurring across a number of shelf areas south of the Antarctic convergence (Kock 2005). Within the Atlantic sector of the Southern Ocean the species is distributed in productive regions across the Scotia Arc, from South Georgia to the Antarctic Peninsula (North 2005). A commercial bottom trawl fishery for the species developed at South 
Georgia in the 1970s, with a peak catch of over $150,000 \mathrm{t}$ in the 1982-83 fishing season (CCAMLR 2012). In the wake of the 1971 collapse of the marbled rockcod (Notothenia rossii) fishery (Trathan and Reid 2009), catches of mackerel icefish were intermittently high as the stock experienced increased exploitation. By the middle of the 1980s commercial catches became increasingly variable and relatively low (Kock 2005). Management efforts by the Commission for the Conservation of Antarctic Marine Living Resources (CCAMLR) to control the exploitation rate and allow the stock to recover extended to a full closure of the demersal fishery in the early 1990s. The fishery re-opened in 1996, but catches have remained at low levels $(<7000 \mathrm{t}$ ) since then (Hill et al. 2005; CCAMLR 2012). Since 2010 a fishery which now utilises pelagic nets towed close to the seabed has been certified by the Marine Stewardship Council (MSC).

Mackerel icefish is known to be an important food source within the food-web at South Georgia, becoming a primary prey species for pelagic predators such as the Antarctic fur seal (Arctocephalus gazella) in years of low krill availability (Agnew et al. 1998). Recent ecosystem modelling suggests that the biomass of mackerel icefish at South Georgia estimated from bottom trawl survey data does not account for the amount of icefish estimated to be required by the local pelagic food-web (Hill et al. 2012). In fact, the model suggests that there is up to an order of magnitude more fish biomass required than the amount estimated from surveys. This discrepancy might suggest an incomplete scientific understanding of mackerel icefish population dynamics from the surveys, or of the species' ecosystem interactions, or some combination of both. In addition, it becomes increasingly difficult to disentangle the effects of the various components of mortality when considered in the context of the recovery of previously exploited upper trophic level predator species (e.g. Antarctic fur seals; Hill et al. 2005; Reid et al. 2005; Wiedenmann et al. 2011). Given that part of the remit of CCAMLR is to ensure rational use of living resources and the maintenance of ecosystem structure, uncertainty in estimates of the size and dynamics of resources should be minimised (Agnew 1997).

Bottom trawl surveys have been carried out intermittently in CCAMLR sub-area 48.3 (Fig. 1 , inset) since the late 1980s. The survey has been recognised as problematic for the estimation of icefish abundance in several respects (Everson et al. 1996). In general, the data from these surveys are characteristic of a patchily distributed stock: with right-skewed statistical distributions, large variance estimates, and often considerable proportions of null observations. These statistical properties can result in mean density estimates of low precision, regardless of whether sampling effort is considered to be adequate (McConnaughey and Conquest 1993). Occasional, relatively high 
(seemingly anomalous) survey catches, sometimes considered as outliers, were in fact treated as being representative unless they were known to be a result of some error in sampling or data recording (Hansen et al. 1983). Outliers in fish survey data are often better described as extreme values (McConnaughey and Conquest 1993). However, these extreme values can render the use of normal probability approximations invalid (Särndal et al. 1978). They may also lead to excessively large imprecise survey indices, making it difficult to detect relative changes in stock size. Choice of abundance estimation method is, therefore, crucial to effective management (Smith 1993; Olsen and Schafer 2001). Consequently, CCAMLR's Working Group on Fish Stock Assessment (WG-FSA) has had concerns about the suitability of the current methods used for assessing the status of this stock (Agnew et al. 1998; Hillary et al. 2010).

Annual quotas for the fishery are derived from intentionally conservative biomass estimates (based on the lower limit of $90 \%$ bootstrapped confidence intervals) as part of a sustainable harvest management plan. It is assumed that mean stock biomass is equal to mean biomass estimated from the survey; i.e. catchability coefficient $q=1$ (Agnew et al. 1998; Hillary et al. 2010). The bootstrapping routine used (Hillary et al. 2010) was adapted from a method used to pool weighted density-at-length data to compensate for low sample sizes within strata (de la Mare and Williams 1996). Although this method is thought to provide adequate estimates of the mean (Hillary et al. 2010), the associated variance and confidence estimates have not been evaluated. Given that the quotas are based on confidence intervals which are potentially invalid, alternative methods for estimating uncertainty around mean density should be explored. Furthermore, given the assumption that $q=1$, it is difficult to explain the discrepancy between these survey-based biomass estimates and the amount of icefish estimated to be required by the local foodweb. It suggests that either the assessment assumption regarding gear selectivity for the species is incorrect (the survey trawl inadequately samples the entire vertical distribution of the stock), or that the food web models are incorrectly parameterised.

Given that quotas for mackerel icefish are based on confidence intervals constructed using the CCAMLR reweighting method, this approach is evaluated here. The objective of this study was to determine the validity of current estimates of mean density of mackerel icefish and the associated uncertainty measures, and to explore alternative methods which might provide valid estimates. The ability of the survey to detect temporal changes in abundance of individual cohorts was also investigated using the length frequency data. This latter analysis was undertaken in an effort to explore the validity of the assumption of $q=1$, and identify evidence of any potential mismatch between species distribution and survey 


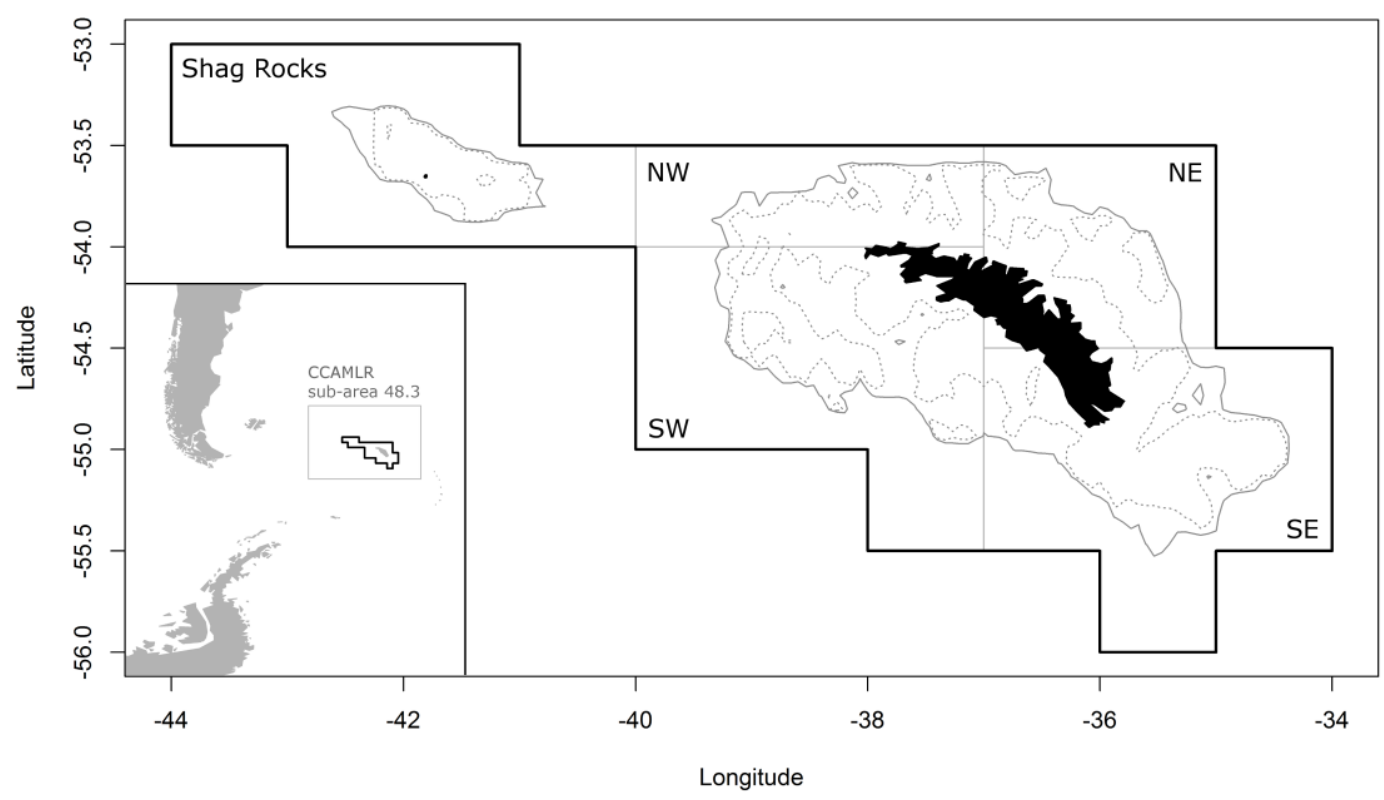

Fig. 1 Map detailing the stratum design of the South Georgia groundfish survey. The light grey polygons represent the survey area divisions: Shag Rocks, Northwest (NW), Northeast (NE), Southeast (SE), and Southwest (SW). The solid line is the $300 \mathrm{~m}$ bathymetric contour; the dotted line is $200 \mathrm{~m}$ bathymetric contour.

method which might lead to an incomplete representation of population dynamics in the stock assessment.

\section{Materials and Methods}

\section{Data sources}

Groundfish survey data were obtained from the Government of South Georgia and South Sandwich Islands (GSGSSI). The GSGSSI database holds records for 20 bottom trawl surveys carried out between 1987 to 2015 (along with a partial survey from 2012 and a radial transect survey from 2003; Table 1). However this analysis will focus only on data collected between 1991 and 2015 when sampling gear and survey methods were standardised. The current survey (Mitchell et al. 2010) follows a stratified random design, across five area strata (Fig. 1), within each of which are two depth strata of $50-200 \mathrm{~m}$ and $>200 \mathrm{~m}$ (generally $<300 \mathrm{~m}$ ). The number of trawl hauls was variable during the earlier part of the time series, but since 2008 a minimum of 70 hauls have been undertaken with sample allocations set for each stratum based on the variability of icefish density observed in previous surveys.

In calculating the area of the seafloor trawled, wingspread was used as opposed to doorspread with the assumption that no herding occurred due to a lack of sand cloud formation (Dickson 1993; L. Featherstone 2012, pers. comm., 6 Dec.). All surveys used an FP120 trawl net, a modified bottom trawl gear without lower wings to minimise damage sustained on rough ground (Parkes 1991). Where measured wingspread values were not available (as was the case in surveys from 2008 
to 2015) a non-linear model was fitted to the available wingspread measurements (Baudron and Fernandes 2014) to estimate wingspread as a function of depth:

$$
\operatorname{Wingspread}(m)=\frac{\alpha * d p(m)}{\beta+d p(m)}
$$

where $d p$ is the mean depth, and $\alpha$ and $B$ are coefficients estimated by non-linear least squares. Haul-level swept-area densities $\left(\mathrm{kg} \mathrm{km}^{-}\right.$ $\left.{ }^{2}\right)$ were calculated given the weight of mackerel icefish caught, distance travelled over ground by the trawl gear, and the wingspread (Saville,
1977).

\section{Density estimation}

Stratified mean density $\left(\bar{d}_{s t}\right)$ and associated variance $\left(s_{s t}^{2}\right)$ were calculated as follows;

$$
\begin{gathered}
\bar{d}_{s t}=\sum_{j=1}^{k} W_{j} \bar{d}_{j} \\
s^{2}{ }_{s t}=\sum_{j=1}^{k} \frac{N_{j}}{N^{2}}\left(N_{j}-n_{j}\right) \frac{s^{2}{ }_{j}}{n_{j}}
\end{gathered}
$$

where $\bar{d}_{j}$ is the estimated mean abundance in stratum $j, W_{j}$ is the proportion of the total survey area in stratum $j, N_{j}$ is the total number

\begin{tabular}{|c|c|c|c|c|}
\hline Year & Number of hauls & $\begin{array}{l}\text { Arithmetic mean swept- } \\
\text { area density }\left(\mathrm{kg} \mathrm{km}^{-2}\right)\end{array}$ & Variance $\left(\mathrm{kg} \mathrm{km}^{-2}\right)$ & Proportion of null hauls \\
\hline 1991 & 78 & 755.1 & $2.22 \times 10^{6}$ & 0.09 \\
\hline 1992 & 82 & 1024 & $2.91 \times 10^{6}$ & 0.06 \\
\hline 1994 & 81 & 479.4 & $7.09 \times 10^{5}$ & 0.05 \\
\hline 1997 & 58 & 2013 & $4.47 \times 10^{7}$ & 0.05 \\
\hline 2000 & 41 & 1255 & $2.79 \times 10^{7}$ & 0.05 \\
\hline 2002 & 63 & 976.9 & $1.10 \times 10^{7}$ & 0.03 \\
\hline 2004 & 65 & 1478 & $2.61 \times 10^{7}$ & 0.25 \\
\hline 2005 & 51 & 124.5 & $6.84 \times 10^{4}$ & 0.39 \\
\hline 2006 & 66 & 2965 & $9.90 \times 10^{7}$ & 0.17 \\
\hline 2007 & 46 & 1840 & $4.59 \times 10^{7}$ & 0.13 \\
\hline 2008 & 70 & 2201 & $7.07 \times 10^{7}$ & 0.11 \\
\hline 2009 & 72 & 1504 & $1.49 \times 10^{7}$ & 0.07 \\
\hline 2010 & 74 & 2195 & $6.68 \times 10^{7}$ & 0.05 \\
\hline 2011 & 84 & 1832 & $2.45 \times 10^{7}$ & 0.05 \\
\hline 2013 & 70 & 10050 & $2.49 \times 10^{9}$ & 0.01 \\
\hline 2015 & 77 & 985.8 & $5.48 \times 10^{6}$ & 0.08 \\
\hline
\end{tabular}

Table 1 Summary of mackerel icefish survey data collected during groundfish surveys carried out at CCAMLR sub-area 48.3 
of possible hauls in stratum $j, N$ is the total number of possible hauls in the survey area, $n_{j}$ is the number of hauls sampled in stratum $j, s^{2}{ }_{j}$ is the estimated variance for stratum $j$, and $k$ is the total number of strata. Although parametric confidence intervals could be constructed for these estimates, this would be with the assumption that survey estimates follow a normal distribution. Small sample sizes in many strata and skewed sample distributions may render those parametric confidence intervals uninformative. In addition, sample variances are not constant across strata, further complicating the application of the Central Limit Theorem (Cochran 1977). So although the mean $\bar{d}_{s t}$ variance $s_{s t}^{2}$ are valid quantities, the estimation of confidence intervals, which are needed for management, is not so straightforward.

Mean icefish density was then calculated in accordance with the methods approved by CCAMLR's WG-FSA (Hillary et al., 2010). Firstly, haul-level density data was reweighted to account for the different sampling intensities in each stratum (de la Mare 1994; de la Mare and Williams 1996).

$$
D_{i}=d_{i, j} \times \frac{A_{j}}{\sum_{j=1}^{k} A_{j}} \times \frac{\sum_{j=1}^{k} n_{j}}{n_{j}}
$$

where $D_{i}$ is the weighted density value for haul $i, d_{i, j}$ is the observed density for haul $i$ in stratum $j$, and $A_{j}$ is the stratum area. Bootstrapped estimates of the mean were then calculated across the entire re-weighted set of density values $D_{i}$. The current CCAMLR assessment incorporates the non-stratified arithmetic mean in a non-parametric bootstrap routine $\left(\bar{D}_{\text {cboot }}\right)$ using random re-sampling with replacement (Efron and Tibshirani 1993).

$$
\bar{D}_{\text {Cboot }}=\frac{1}{B} \sum_{b=1}^{B} \frac{1}{n_{b}} \sum_{i=1}^{n_{b}} D_{i}
$$

where $B$ is the total number of bootstrap replicates, and $n_{b}$ is the number of hauls in bootstrap sample $b$. The variance $\left(s_{\text {cboot }}^{2}\right)$ may similarly be derived given the arithmetic mean $\bar{D}_{\text {Cboot }}$ :

$$
s_{\text {cboot }}^{2}=\frac{1}{B} \sum_{b=1}^{B} \frac{1}{n_{b}} \sum_{i=1}^{n_{b}}\left(\frac{1}{n_{b}} \sum_{i=1}^{n_{b}} D_{i}-D_{i}\right)^{2}
$$

For the assessment, confidence limits around the mean estimates $\bar{D}_{\text {cboot }}$ are simply then taken from the appropriate percentiles of the bootstrap distribution of mean estimates, and the same method was applied here to construct $95 \%$ confidence intervals.

Finally stratified naïve $\left(\bar{d}_{\text {Nboot }}\right)$ and rescaling $\left(\bar{d}_{R b o o t}\right)$ bootstrap estimators of the mean $\left(\bar{d}_{s t}\right)$ were also applied to the observed icefish density data $d_{i, j}$ (see Smith 1997 for details). The naïve bootstrap involves resampling observations $d_{i, j}$ with replacement within each stratum $n_{j}^{*}$ times, calculating a mean $\vec{d}_{j}^{*}$ at each resampling. The stratified mean may then be calculated as:

$$
\bar{d}_{s t, b}=\sum W_{j} \bar{d}_{j}^{*}
$$


This process is repeated $B$ times and the bootstrap estimates of the mean ( $\mathrm{Eq} 8$ ) and variance (Eq 9) are given by:

$$
\begin{gathered}
\bar{d}_{\text {Nboot }}=\sum_{b=1}^{B} \bar{d}_{s t, b} /_{B} \\
s^{2}{ }_{\text {Nboot }}=\frac{1}{B-1} \sum_{b=1}^{B}\left(\bar{d}_{s t, b}-\bar{d}_{\text {Nboot }}\right)^{2}
\end{gathered}
$$

The rescaling bootstrap estimator was formulated to provide unbiased estimates of variance as well as mean. A random sample of size $m_{j}\left(<n_{j}^{*}\right)$ with replacement is taken from the samples in stratum $j\left(d^{\bullet}{ }_{j x}, x=1, \ldots, m_{j}\right)$. The following calculations are then performed:

$$
\begin{gathered}
\tilde{d}_{j x}=\bar{d}_{j}+\left(\frac{m_{j}\left(1-f_{j}\right)}{\left(n^{*}{ }_{j}-1\right)}\right)^{1 / 2}\left(d^{\cdot}{ }_{j x}-\bar{d}_{j}\right) \\
\tilde{d}_{j}=m_{j}{ }^{-1} \sum_{x=1}^{m_{j}} \tilde{d}_{j x} \\
\tilde{d}_{s t, b}=\sum_{j=1}^{k} W_{j} \tilde{d}_{j}
\end{gathered}
$$

The process in Eq 10, 11 \& 12 is repeated $B$ times, and the estimates of mean and variance are calculated as in Eq 8 \& 9, substituting $\tilde{d}_{j}$ for $\bar{d}_{s t, b}$. Percentile confidence limits were then constructed for the estimates of the mean (Efron and Tibshirani 1993). These intervals do not require any distributional assumptions, assuming instead that the observed samples are representative of the distribution of the underlying population. Estimates for each bootstrapping routine were then compared to the standard arithmetic estimators (Eq $2 \& 3$ ) to test their relative performance. Behaviour of variance estimates in terms of percentage deviation from the stratified arithmetic variance as a function of the number of bootstrap replicates was also examined, as agreement between bootstrap variance estimates $\left(s^{2}{ }_{N b o o t}\right.$ and $\left.s^{2}{ }_{R b o o t}\right)$ and $s^{2}{ }_{s t}$ would affirm the validity of bootstrap percentile confidence limits (i.e. for the bootstrap confidence limits to be considered valid and useful, there should be good agreement between the bootstrap estimates of mean and variance and the unbiased arithmetic estimators).

\section{Consistency of stock demographics in survey} data

Mean length frequency distributions were calculated for each survey year, given length data collected from sub-samples of the catch at a number of trawl stations, and the corresponding total icefish catch weights. Numbers-at-length were raised to haul level according to the ratio of sub-sample weight to haul weight, corrected for differences in sampling intensity in different strata (see Eq 4). Whole-survey estimates of proportions at each $1 \mathrm{~cm}$ length class were then calculated. Mean numbers-at-length were calculated by applying these proportions in turn to the estimates of mean swept-area density, given a surveyspecific length-weight relationship, $W=a L^{b}$, where $W$ is the weight $(\mathrm{g}), L$ is the length $(\mathrm{cm})$, and $a$ and $b$ are fitted constants (Online Resource 1, Fig. S1). Finite mixture distribution models were fitted to each estimate of mean 
numbers-at-length using an expectation maximisation algorithm, specifying starting values of the expected mean lengths-at-age as derived from the Von Bertalanffy growth parameters used in the assessment (Online Resource 1, Fig. S2; Macdonald and Pitcher 1979; Benaglia et al. 2009; Hillary et al. 2010). It was assumed that each distribution in the mixture corresponded to a given age class within the population, and thus numbers-at-age could be estimated according to the probability of an individual of a particular size belonging to a given cohort. Log-transformed numbers-atage data for each cohort were plotted to assess the assumption of $q=1$, and the consistency of the survey in tracking cohorts through time (Cotter et al. 2007).

\section{Results}

The largest values of $d_{i, j}$ tended to occur to the northwest of the main South Georgia shelf, and off Shag Rocks (Fig. 2). Areas of rougher ground directly to the south of the island have been sparsely sampled to minimise time lost to gear replacement and repair: when these areas have been sampled they have yielded low densities. Relatively low densities were also encountered to the southwest and east of the island. These trends in spatial distribution of icefish density are likely to be driven by food (specifically krill) availability (Frolkina 2002). Approximately 15\% of null observations across all surveys were encountered at depths between 100 and $200 \mathrm{~m}$, where $49.8 \%$ of total hauls were conducted within this depth range. The remaining null hauls were observed at depths greater than 200 $\mathrm{m}$. The majority of this remainder, $50 \%$ of all null hauls, were recorded below the $300 \mathrm{~m}$ depth contour where just over $10 \%$ of all hauls were conducted. This suggests a tendency for the species to aggregate in shallower waters within the bounds of the shelf edge. The overall proportion of null observations also varied temporally, from as low as 0.01 up to 0.39 in 2005. 


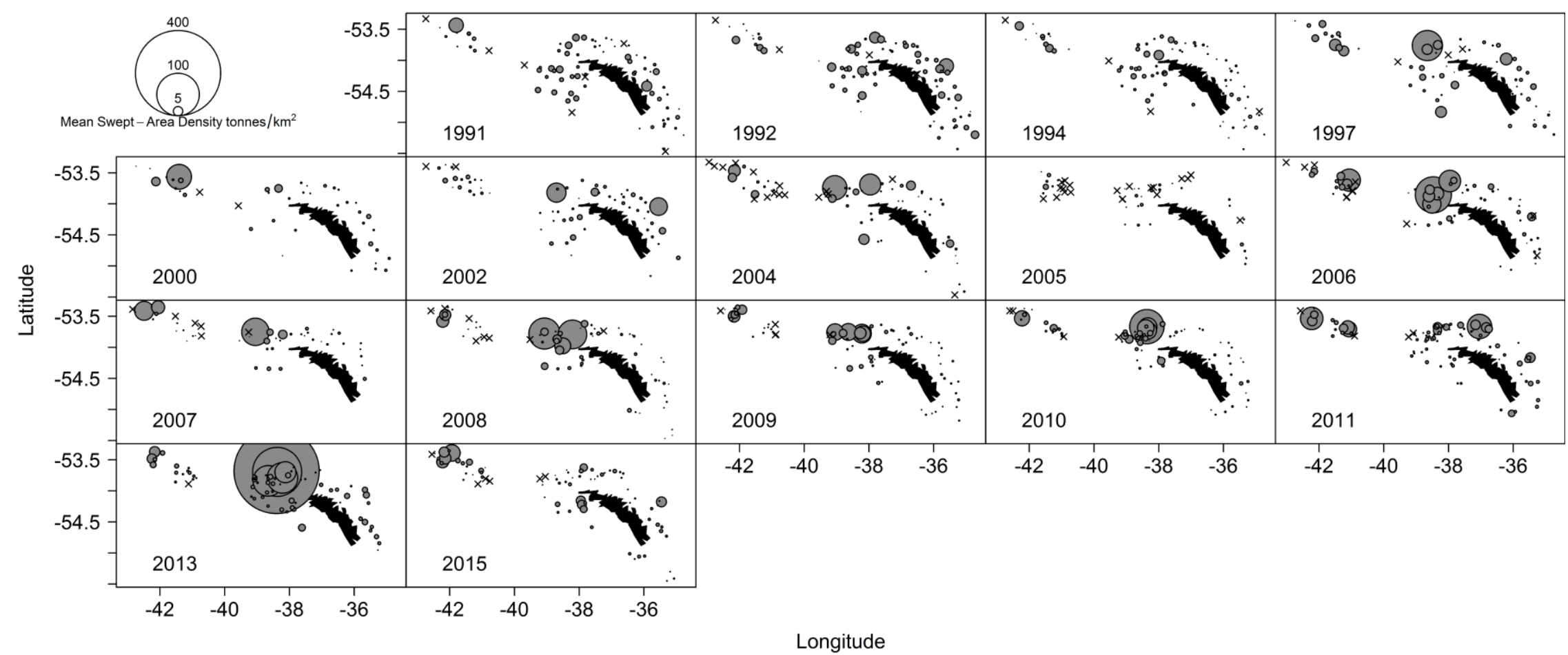

Fig. 2 Maps showing spatial distribution of haul level swept-area densities $\left(\mathrm{kg} \mathrm{m}^{-2}\right)$ derived from the UK South Georgia Groundfish survey data. Each haul is represented by a circle, the radius of which is relative to the swept-area density. Null observations are represented by an ' $x$ '. 


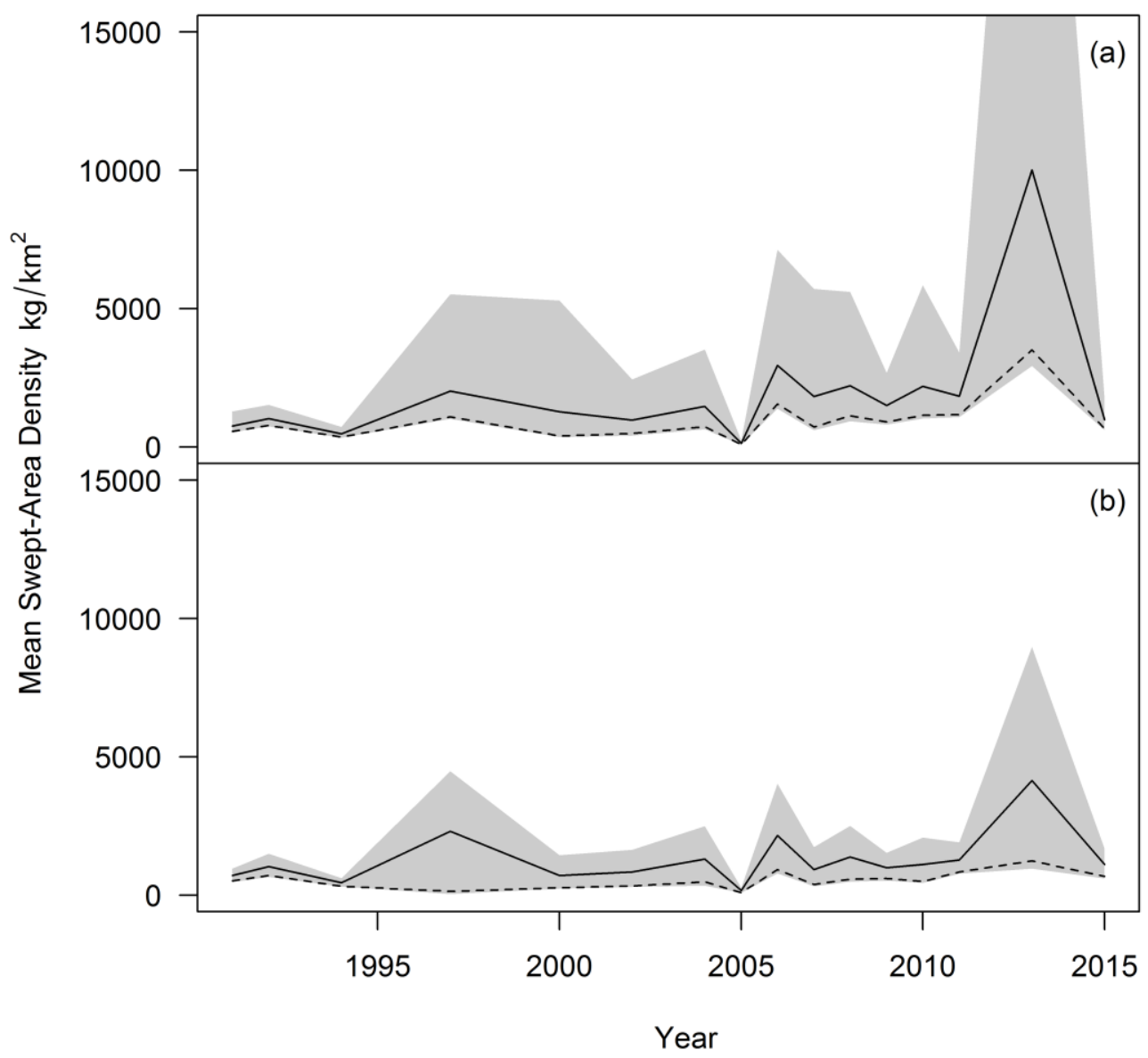

Fig. 3 Mean density estimates $\left(\mathrm{kg} \mathrm{km}^{-2}\right)$ of C. gunnari from the UK South Georgia groundfish survey from 1991 to present. Panel (a) shows estimates using the current method with percentile confidence intervals, and panel (b) shows the alternative method which best approximated the standard stratified estimators, the rescaling bootstrap, with percentile confidence intervals. The dotted black lines show the $5^{\text {th }}$ percentile used as an estimate of mean biomass in the assessment.

The different time series of density estimates followed similar trends (Fig. 3; Online Resource 1, Fig. S3). Estimates based on the CCAMLR method, $\bar{D}_{\text {Cboot, }}$, were marginally higher than all other estimates, and substantially higher in 2013 when an extremely large catch inflated the estimate of the mean. Variance estimates $s^{2}{ }_{\text {Rboot }}$ best approximated $s^{2}$, and so the associated $\bar{d}_{R b o o t}$ estimates were deemed the most appropriate series to take forward to the mixture distribution analysis (Fig. 4). $s^{2}$ cboot were positively biased (relative to $s_{s t}^{2}$ ) by several orders of magnitude, raising further doubts regarding the validity of the CCAMLR method. The negative bias associated with $s^{2}{ }_{\text {bboot }}$ as observed by Smith (1997) was also present here, and so this method was not considered further. Bootstrap confidence limits around mean estimates followed approximately the same trends, although the percentile method used for $\bar{D}_{\text {cboot }}$ provided generally wider confidence limits (Fig. 3). Where relatively low catch sizes were recorded across an entire 
survey (leading to low mean density estimates; e.g. 1994 and 2005) there was less inter-haul variability and thus narrower confidence limits than other years. The proportion of null observations was also greatest in 2005, which is similarly reflected in relatively low mean density estimates for that year across all estimators (Fig. 3; Online Resource 1, Fig. S3).

The exception to the broad similarities in trends in density estimates occurred where a relatively extremely high density haul was recorded, specifically in the 2013 survey. In this survey the $\bar{d}_{R b o o t}$ estimate was lower than that of $\bar{D}_{\text {Cboot }}$ by $>5700 \mathrm{~kg} \mathrm{~km}^{-2}$ (Fig. 3). Confidence limits around $\bar{D}_{\text {Cboot }}$ for 2013 were wider than those around the corresponding values of $\bar{d}_{R b o o t}$. The 2013 mean density estimate was substantially lower when using $\bar{d}_{R b o o t}$, despite the presence of these relatively large hauls. In this case the stratified rescaling bootstrap estimator weights the influence of the two largest hauls $\left(>39,000\right.$ and $\left.>10,000 \mathrm{~kg} \mathrm{~km}^{2}\right)$ on $\bar{d}_{R b o o t}$ according to the sampling fraction of the northwest stratum and its area as a proportion of the entire survey, resulting in estimates which are lower than $\bar{D}_{\text {cboot }}$. The time series of $\bar{d}_{R b o o t}$ estimates was identified as providing the best basis for an index of relative abundance of mackerel icefish out of those tested, as this method best approximated the results from standard stratified mean and variance estimators, with substantially less uncertainty than is associated with the current method (Fig. 3 \& 4; Online Resource 1, Fig. S3). Confidence intervals around $\bar{D}_{\text {cboot }}$ were wider than those around $\bar{d}_{R b o o t}$ in 14 out of 16 survey estimates. The exceptions to this were the 1992 and 2005 surveys in which estimates were relatively low and confidence bounds relatively narrow for both estimators.

Assuming a population is in equilibrium, annual survival rates should be approximately constant, with numbers-at-age decreasing geometrically according to various components of mortality (Chapman and Robson 1960). Logtransformed numbers-at-age for each cohort or

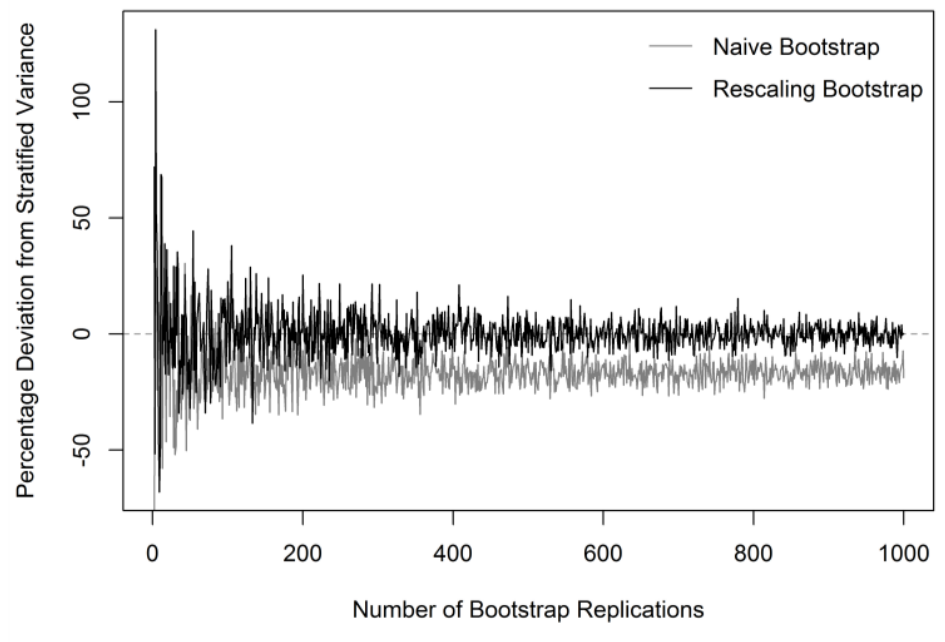

Fig. 4 Performance of variance estimates from 2015 survey data for the naïve and rescaled bootstrap as a function of the number of bootstrap replicates. Mean percent deviation for variance estimates using the CCAMLR bootstrap method was 93045. 


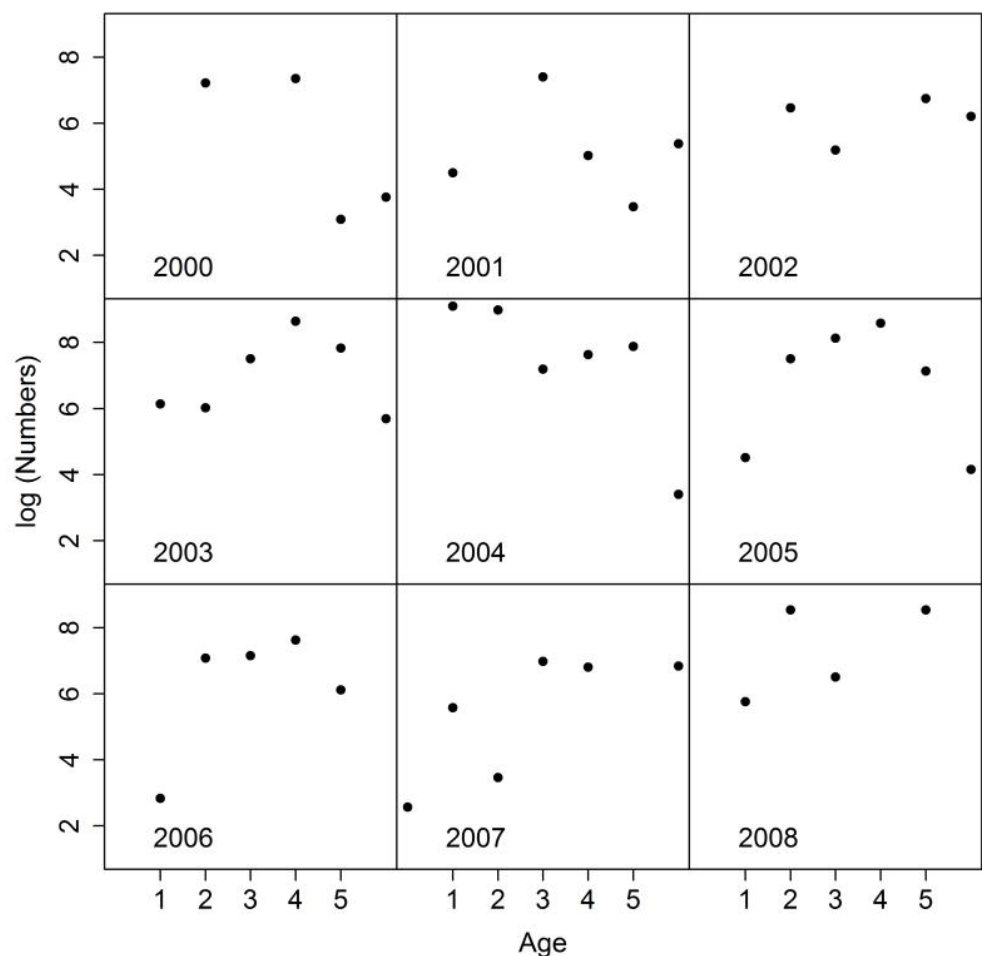

Fig. 5 Log-transformed mean numbers-at-age estimated from mixture distribution analysis of numbers-at-length per $\mathrm{m}^{-2}$ data for cohorts (year of birth indicated in each panel) of icefish which could be tracked annually across a reasonable range of years. Cohort year is indicated in the top right of each panel. year class (year-class curves, Fig. 5) indicate a poor detection of any clear trends in numbersat-age through time. These trends would follow a descending straight line relationship across age classes if all fish were fully selected by the fishing gear, sampling were unbiased, and the total mortality rate within the population was independent of age and survey year. Typically, in survey data, there is some selection on younger ages, or poor juvenile availability to the survey, producing a characteristic year-class curve "hook". In some cases, as with the 2003 and 2005 cohorts, there is a reasonable approximation of the latter: there are low numbers at first, increasing to a peak at age three, and decreasing thereafter. This is not in agreement with the assumption held by the assessment of full selectivity across age classes. This assumption would lead one to expect the highest observed numbers in the youngest age class, decreasing geometrically with increasing age due to mortality (Hillary et al. 2010). However, the estimated numbers-at-age across all surveys do not follow this expected form, introducing doubt as to the validity of that assumption. As the relationship between abundance and age does not appear to be consistent (or indeed obvious) from cohort-tocohort, some degree of unexplained error in terms of mean numbers-at-age across all age classes in all surveys seems to have been introduced. The 2002 cohort was not detected in the 2006 survey although it was present again in the 2007 and 2008 data. This also suggests that error may be introduced through the sampling method where some portion of the stock is not being represented in the data. Age zero individuals appear to have only been detected in the 2007 survey; this is perhaps a 
function of that survey being conducted later than usual (September) in that year.

\section{Discussion}

The stratified re-scaling bootstrap estimators $\left(\bar{d}_{R b o o t}\right.$ and $\left.s^{2}{ }_{R b o o t}\right)$ were found to be the equivalent to $\bar{d}_{s t}$ and $s_{s t}^{2}$, and thus their associated bootstrap confidence intervals offer an appropriate measure of uncertainty for abundance estimates of mackerel icefish at South Georgia. The proportion of null observations varies quite substantially from year-to-year, but regardless of the degree of zero inflation, the bootstrap simply assumes that null hauls are a natural part of the underlying population distribution (Efron and Tibshirani 1993; Smith 1997). In a simplified sense, these null trawls represent the amount of area surveyed which is unoccupied habitat, and directly affects the calculation mean estimates. This concept has its biological limitations as it implies that the species of interest has limited mobility. Perhaps, given the tendency of null observations to be recorded near the shelf edge, and the presence of larger hauls in deeper waters in years of higher mean density (e.g. 1997 and 2013), they may represent some form of density dependent range contraction whereby deeper waters become occupied with increased abundance. It should also be kept in mind that mackerel icefish are known to undergo vertical and horizontal migrations, suggesting that there could be a number of reasons for an apparent absence from a given area (Kock and Everson 1997; Frolkina 2002). A typical stratified random sampling approach involves combining mean estimates and uncertainty measures from all strata. Confidence limits constructed around the standard estimates do, however, require large sample sizes for the application of the Central Limit Theorem (Smith 1997), which is not met by the South Georgia icefish data. Small sample sizes observed in some strata are compensated for to some degree by the use of CCAMLR's weighted pooled bootstrapping method, although exceptionally large hauls (such as that encountered in 2013) still affect precision substantially. Crucially, the CCAMLR method is applied without regard for the survey design, and strata with small sample sizes are likely to be underrepresented in final estimates. The rescaling method deals with these problems through re-sampling within each stratum, and the application of stratum weightings. The fact that the survey may suffer from some degree of over-stratification also remains.

The mixture distribution estimates of mean numbers-at-age indicate that at least some of the cohorts are poorly detected across all surveys (Fig. 5). Although some degree of variability in the ability of the sampling method to detect cohorts through time is expected, data which is too noisy to interpret and an apparent lack of detection of some cohorts is cause for concern given the assumptions of the assessment. Estimates of abundance are biased to some degree because of this sampling error. 


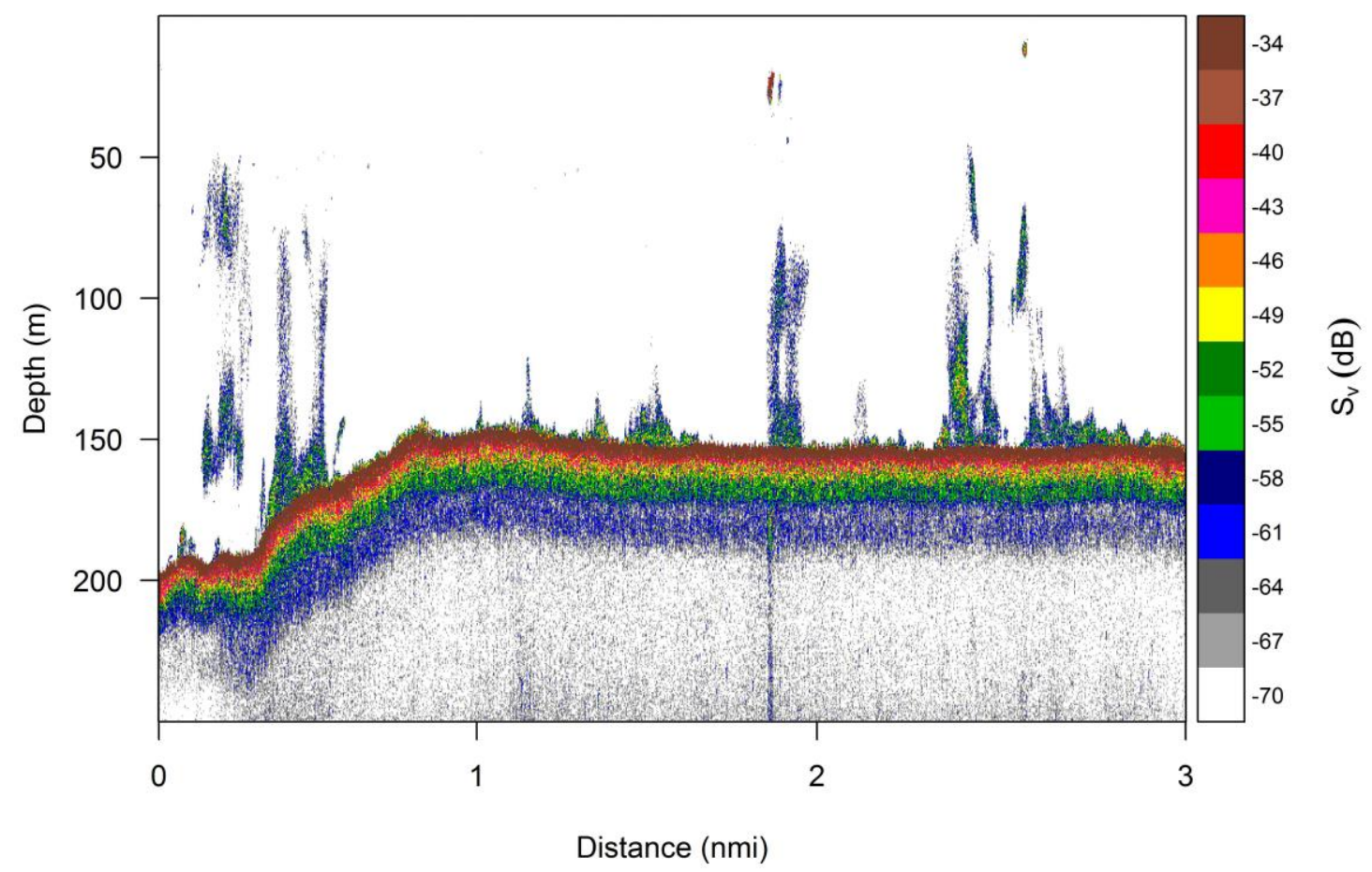

Fig. 6 Scrutinized echogram from the 2006 survey showing echotraces of mackerel icefish recorded using a Simrad EK500 echosounder at $70 \mathrm{kHz}$. A homogenous catch of $50 \mathrm{~kg}$ of mackerel icefish (ranging in size from $>20-35 \mathrm{~cm}$ ) was obtained by trawling on these marks at depths of approximately $60-140 \mathrm{~m}$.

Although basing quotas on conservative abundance estimates is precautionary by definition, it would be preferable to have an internally consistent survey, yielding a more realistic representation of population dynamics. As the commercial fishery utilises pelagic gears which are typically towed close to the seabed, it is potentially selecting a specific demographic component(s) of the stock. Any identifiable pattern in vertical distribution would, therefore, be invaluable in determining what portion of the population is vulnerable to the fishing gear and ultimately fishing pressure. Attempts have been made to address this problem by restricting survey sampling to daylight hours in order to reduce bias thought to be brought about by diurnal migrations undertaken by the species (Everson et al. 1999). However, cursory examination of acoustic data collected in more recent years indicates that this is not an adequate solution to the problem. For instance, in 2006, substantial pelagic aggregations were detected acoustically, from which a sample of $50 \mathrm{~kg}$ of mackerel icefish was obtained, while fishing between 60 and $140 \mathrm{~m}$ depth (between 20-100 $\mathrm{m}$ off the seabed), at approximately 15:00 hrs (Fig. 6). These acoustic data merit further investigation to determine whether the amount of fish unavailable to the demersal trawl has a significant effect on abundance estimates. This proportion could be used to introduce a correction factor for trawl survey estimates. The assumption of no herding is a potential source of further uncertainty. 
Mackerel icefish behaviour around fishing gear is poorly understood, and although there may be no sand cloud formation, other stimuli such as trawl doors in contact with the seabed and short, bouncing trawl bridles may elicit a behavioural response and generate bias (Glass and Wardle 1989, L. Featherstone 2014, pers. comm., 21 Mar.).

The quality of survey information becomes increasingly important when uncertainties exist around the trophic structure of the ecosystem within which the fishery operates. In this context, the South Georgia icefish stock has largely unquantified and poorly understood pressures exerted on it - directly and indirectly - as a result of the dynamics of various components of the local food web (Hill et al. 2012). Krill is an abundant and important (keystone) prey species in the Southern Ocean and the South Georgia shelf retains relatively high densities of krill after they have been advected across the Scotia Sea and Weddell Sea (Tarling and Cuzin-Roudy 2007; Murphy et al. 2013). In addition to the apparent inter-annual changes in the availability of krill, longer-term monitoring of the species suggests a declining trend in krill abundance across the Southern Ocean (Atkinson et al. 2004; Trathan et al. 2012). Fur seals and macaroni penguins (Eudyptes chrysolophus) are both highly efficient predators of krill, which also demonstrate adaptive foraging strategies based on fluctuations in local availability (Waluda et al. 2009). Competition for food between these predators is likely to have increased in recent decades due to the continued recovery of fur seal populations following their near extirpation from the region over a century ago (Reid et al. 2005; Hill et al. 2006). When krill is scarce, mackerel icefish assumes a role of primary importance in the diet of these and other pelagic predators (Agnew et al. 1998). Due to these flexible foraging behaviours, and the estimated biomass of prey required to sustain ever-increasing predator populations, the associated natural mortalities incurred by icefish stocks could be considerable (Hill et al. 2012). This interchange of trophic importance between krill and icefish is further complicated by the fact that icefish is itself a significant predator of krill (Main et al. 2008). Other predators which are believed to be increasing in numbers following the cessation of intensive exploitation include krill-feeding whales (Wiedenmann et al. 2011). Such large, mobile cetaceans will exert substantial pressure on zooplankton resources, which may cause less specialised predators to target icefish as an alternative to krill in response to increasing competition (Hill et al. 2005).

Conventionally, catch limits are based on the best estimates of stock size. A precautionary approach would then be applied by setting limits based on the uncertainty in abundance estimates, and when those limits are reached an agreed set of actions is undertaken in order to avoid irreversible damage to the stock (Caddy 2002). It would be advisable to 
adopt that type of standard approach as opposed to incorporating the uncertainty into the estimates themselves as is the practice here, in anticipation of meeting future MSC certification requirements. Despite the intentionally conservative nature of setting quotas based on lower quantile biomass estimates of icefish, and any inherent underestimation bias which may exist due to sampling method, it remains preferable to maximise accuracy through the use of valid estimates. The more accurate estimates of icefish abundance become, the more reliable information will be regarding relative changes in the size of the population. Whether the longterm decline in catches of mackerel icefish is representative of an anthropogenic stock collapse or a suppression of population growth due to predator-prey interactions has been debated for some time (Everson et al. 1999). The status of mackerel icefish at South Georgia is often expressed in terms of potential for the stock to "recover" to the levels which yielded historically high catches (Hill et al. 2005; Reid et al. 2005; Trathan et al. 2012). Due to changes in fishing practices, reporting and regulations which have occurred in the last 50 years it is difficult to determine how much of the apparent downward trajectory of the stock can be explained by changes in fishing intensity. Data relevant to this key issue simply does not exist for the early years of the fishery, making attempts to interpret catch figures as changes in abundance futile. In terms of the data available,
1987 may be a key year in understanding longer term trends in the size of the population. In that year, commercial catches in excess of $80,000 \mathrm{t}$ were recorded whereas the survey biomass estimate for the same season was approximately 40,000 t (Everson et al. 1999; CCAMLR 2012). Although this disparity between survey estimates and observed catch rates is exaggerated due to the precautionary nature of management, a more comprehensive assessment is required to better understand trends in abundance. Perhaps instead of considering survey results in terms of recovery to some former ideal, focus could be shifted to uncertainties around the current status of the population and the dynamic role of mackerel icefish in the local food-web. With the numerous interacting variables which affect the population in mind, adopting a more appropriate survey design and analysis would be a step towards precautionary yet optimised assessment to suit all stakeholders.

Assessment of the stock could benefit from the development of a survey which would account for any variability in availability of icefish to bottom trawl sampling. Given the difficulty in a priori prediction of locations of high density aggregations, trawling in strictly predetermined locations does not necessarily always make sense. An adaptive design could be considered whereby effort may be focussed on a particular area(s) where extreme densities are encountered. Depending then on the level of confidence in how representative the 
sampling programme is, an adaptive model could be implemented to account for presence/absence, low density and high density areas (Stefánsson 1996; Thorson and Stewart 2011). An element of acoustic data analysis, particularly in high density areas, could be included to determine vertical distribution of the stock (e.g. Northwest Atlantic cod; Mcquinn et al. 2005), and echosounder data is already routinely collected on survey (Fig. 6). Ideally, given that mackerel icefish do not possess a swim bladder, collection of data across as many frequencies as possible would improve target discrimination which would provide qualitative data on the size and extent of dense aggregations of icefish (Everson et al. 1996). There is also potential to utilise acoustic data collected concurrently with trawl events to investigate the extent of herding for the improvement of abundance estimates (von Szalay et al. 2007). If knowledge of the target strength of the species were improved, acoustic methods could be extended to the estimation of abundance of the pelagic component of the stock (Simmonds and MacLennan 2005).

\section{Conclusions}

The stratified rescaling bootstrap-mean estimator was found to be the most appropriate method of those tested for constructing confidence intervals for estimates of density of mackerel icefish. The method yielded comparable estimates of mean and variance to those obtained with the standard stratified estimators, while giving useful and valid uncertainty measures in the form of percentile confidence intervals.

The demographic information coming from the survey is difficult to interpret due to some combination of measurement error arising from variable availability of mackerel icefish to demersal trawl sampling and unequal selectivity of the sampling gear across age classes. The resultant series of estimates of numbers-at-age is noisy, and difficult to interpret. The survey would benefit from the incorporation of acoustic data in the estimation of abundance, as well as an in-depth investigation of gear selectivity.

\section{Acknowledgments}

The authors wish to thank the crews, fishermen and scientists who conducted the various surveys from which data were obtained, and Mark Belchier and Simeon Hill for their contributions. This work was supported by the Government of South Georgia and South Sandwich Islands. Additional logistical support provided by The South Atlantic Environmental Research Institute, with thanks to Paul Brickle. Thanks to Stephen Smith of Fisheries and Oceans Canada (DFO) for help in constructing bootstrap confidence limits. Paul Fernandes receives funding from the MASTS pooling initiative (The Marine Alliance for Science and Technology for Scotland), and their support is gratefully acknowledged. MASTS is funded by the Scottish Funding Council (grant reference HR09011) and contributing institutions. We also wish to thank two anonymous referees for their helpful suggestions on earlier versions of this manuscript. 


\section{References}

Agnew D (1997) Review-The CCAMLR Ecosystem Monitoring Programme. Antarct Sci 9:235-242

Agnew D, Everson I, Kirkwood G, Parkes G (1998) Towards the development of a management plan for the mackerel icefish (Champsocephalus gunnari) in Subarea 48.3. CCAMLR Sci 5:63-77

Atkinson A, Siegel V, Pakhomov E, Rothery P (2004) Longterm decline in krill stock and increase in salps within the Southern Ocean. Nature 432:100-103

Baudron AR, Fernandes PG (2014) Adverse consequences of stock recovery: European hake, a new "choke" species under a discard ban? Fish Fish. doi: 10.1111/faf.12079

Benaglia T, Chauveau D, Hunter DR, Young DS (2009) mixtools: An $\mathrm{R}$ package for analyzing finite mixture models. J Stat Softw 32:1-29

Caddy JF (2002) Limit reference points, traffic lights, and holistic approaches to fisheries management with minimal stock assessment input. Fish Res 56:133-137

CCAMLR (2012) Report of the Working Group on Fish Stock Assessment. In: Report of the Thirty-first Meeting of the Scientific Committee (SC-CAMLR-XXXI), Annex 7, Appendix G. Hobart, Australia

Chapman D, Robson D (1960) The analysis of a catch curve. Biometrics 16:354-368

Cochran WG (1977) Sampling Techniques, Third. John Wiley \& Sons, New York

Cotter A, Mesnil B, Piet G (2007) Estimating stock parameters from trawl cpue-at-age series using yearclass curves. ICES J Mar Sci 64:234-247

Dickson W (1993) Estimation of the capture efficiency of trawl gear. I: Development of a theoretical model. Fish Res 16:239-253

Efron B, Tibshirani RJ (1993) An Introduction to the Bootstrap, First. Chapman \& Hall, New York

Everson I, Bravington M, Goss C (1996) A combined acoustic and trawl survey for efficiently estimating fish abundance. Fish Res 26:75-91

Everson I, Parkes G, Kock K-H, Boyd IL (1999) Variation in standing stock of the mackerel icefish Champsocephalus gunnari at South Georgia. J Appl Ecol 36:591-603

Frolkina Z (2002) Distribution of mackerel icefish (Champsocephalus gunnari)(Channichthyidae) around South Georgia at various stages of its life cycle. CCAMLR Sci 9:49-69

Glass C, Wardle C (1989) Comparison of the reactions of fish to a trawl gear, at high and low light intensities. Fish Res 7:249-266

Hansen M, Madow W, Tepping B (1983) An evaluation of model-dependent and probability-sampling inferences in sample surveys. J Am Stat Assoc 78:776-793

Hill S, Reid K, North A (2005) Recruitment of mackerel icefish (Champsocephalus gunnari) at South Georgia indicated by predator diets and its relationship with sea surface temperature. Can J Fish Aquat Sci 27:25302537

Hill SL, Keeble K, Atkinson A, Murphy EJ (2012) A foodweb model to explore uncertainties in the South Georgia shelf pelagic ecosystem. Deep Sea Res Part II Top Stud Oceanogr 59-60:237-252
Hill SL, Murphy EJ, Reid K, et al. (2006) Modelling Southern Ocean ecosystems: krill, the food-web, and the impacts of harvesting. Biol Rev Camb Philos Soc 81:581-608

Hillary R, Edwards C, Mitchell R, Agnew DJ (2010) Lengthbased assessment for mackerel icefish (Champsocephalus gunnari) at South Georgia. CCAMLR Sci 17:129-137

Kock K, Everson I (1997) Biology and ecology of mackerel icefish, Champsocephalus gunnari: An Antarctic fish lacking hemoglobin. Comp Biochem Physiol Part A Physiol 118:1067-1077

Kock K-H (2005) Antarctic icefishes (Channichthyidae): a unique family of fishes. A review, Part I. Polar Biol 28:862-895

De la Mare WK (1994) Estimating confidence intervals for fish stock abundance estimates from trawl surveys. CCAMLR Sci 1:203-207

De la Mare WK, Williams R (1996) Estimates of absolute recruitment for patagonian toothfish (Dissostichus eleginoides) around Heard Island

Macdonald P, Pitcher T (1979) Age-groups from sizefrequency data: a versatile and efficient method of analyzing distribution mixtures. J Fish Board Canada 36:987-1001

Main CE, Collins MA, Mitchell R, Belchier M (2008) Identifying patterns in the diet of mackerel icefish (Champsocephalus gunnari) at South Georgia using bootstrapped confidence intervals of a dietary index. Polar Biol 32:569-581

McConnaughey R, Conquest L (1993) Trawl survey estimation using a comparative approach based on lognormal theory. Fish Bull 91:107-118

Mcquinn I, Simard Y, Stroud T, et al. (2005) An adaptive, integrated "acoustic-trawl" survey design for Atlantic cod (Gadus morhua) with estimation of the acoustic and trawl dead zones. ICES J Mar Sci 62:93-106

Mitchell RE, Belchier M, Gregory S, et al. (2010) Results of the groundfish survey carried out in CCAMLR sub-area 48. 3 in January 2010. CCAMLR WG-FSA-10/38 38

Murphy EJ, Hofmann EE, Watkins JL, et al. (2013) Comparison of the structure and function of Southern Ocean regional ecosystems: The Antarctic Peninsula and South Georgia. J Mar Syst 109-110:22-42

North AW (2005) Mackerel icefish size and age differences and long-term change at South Georgia and Shag Rocks. J Fish Biol 67:1666-1685

Olsen MK, Schafer JL (2001) A Two-Part Random-Effects Model for Semicontinuous Longitudinal Data. J Am Stat Assoc 96:730-745

Parkes G (1991) The UK fish stock assessment survey bottom trawl for South Georgia. CCAMLR WG-FSA91/16

Pennington M (1996) Estimating the mean and variance from highly skewed marine data. Fish Bull 498-505

Reid K, Hill SL, Diniz TCD, Collins MA (2005) Mackerel icefish Champsocephalus gunnari in the diet of upper trophic level predators at South Georgia: implications for fisheries management. Mar Ecol Prog Ser 305:153161

Särndal C, Thomsen I, Hoem J (1978) Design-Based and Model-Based Inference in Survey Sampling. Scand J Stat 5:27-52 
Saville A (1977) Survey methods of appraising fishery resources. Doc. Tech. FAO sur les Pech. 171

Simmonds E, MacLennan D (2005) Fisheries Acoustics. Theory and Practice., Second Edi. Blackwell Publishing Ltd, Oxford, UK

Smith P (1993) Balancing sampling precision and fisheries management objectives: minimal methods. Bull Mar Sci 53:930-935

Smith SJ (1997) Bootstrap confidence limits for groundfish trawl survey estimates of mean abundance. Can J Fish Aquat Sci 54:616-630

Stefánsson G (1996) Analysis of groundfish survey abundance data: combining the GLM and delta approaches. ICES J Mar Sci 53:577-588

Von Szalay PG, Somerton DA, Kotwicki S (2007) Correlating trawl and acoustic data in the eastern Bering Sea: A first step toward improving biomass estimates of walleye pollock (Theragra chalcogramma) and Pacific cod (Gadus macrocephalus)? Fish Res 86:77-83

Tarling G, Cuzin-Roudy J (2007) Recruitment of Antarctic krill Euphausia superba in the South Georgia region: adult fecundity and the fate of larvae. Mar Ecol Prog Ser 331:161-179

Thorson J, Stewart I (2011) Accounting for fish shoals in single-and multi-species survey data using mixture distribution models. Can J Fish Aquat Sci 68:1681-1693

Trathan P, Reid K (2009) Exploitation of the marine ecosystem in the sub-Antarctic: historical impacts and current consequences. Pap Proc R Soc Tasmania 143:914

Trathan PN, Ratcliffe N, Masden E a. (2012) Ecological drivers of change at South Georgia: the krill surplus, or climate variability. Ecography (Cop) 35:983-993

Waluda CM, Collins MA, Black AD, et al. (2009) Linking predator and prey behaviour: contrasts between Antarctic fur seals and macaroni penguins at South Georgia. Mar Biol 157:99-112

Wiedenmann J, Cresswell KA, Goldbogen J, et al. (2011) Exploring the effects of reductions in krill biomass in the Southern Ocean on blue whales using a statedependent foraging model. Ecol Modell 222:33663379 